\title{
EXPLANATION OF THE TERM FOURTH DIMENSION.
}

\author{
By G. A. Miller, \\ University of Illinois.
}

The term, fourth dimension, should be used in such a way as to be in accord with the common usage of the terms one, two, and three dimensions. Hence we shall first inquire how the latter terms are generally used. All agree that the straight line has only one dimension if the point is regarded as the element. The number of points on a straight line is evidently equal to the number of line segments which lie on this line and have a common end point. Hence a straight line has also only one dimension when these line segments are the elements. On the contrary, a straight line has two dimensions if the line segments on it are regarded as the elements. The truth of this statement will appear more clearly in what follows. It appears, however, very evident that there are more line segments than points on a straight line, since we can establish a one to one correspondence between all these points and the segments which have a common end-point. In this one to one correspondence the segment of zero length may be said to correspond to the common endpoint.

The points on a line are commonly placed in a one to one correspondence with all the real numbers by means of an arrangement in which the numbers represent the distances of the points from a fixed point. Such an arrangement is suggested in the following diagram:

\begin{tabular}{ccccccccc}
\hline-4 & -3 & -2 & -1 & 0 & 1 & 2 & 3 & 4 \\
& & & Fig. 1 & & & &
\end{tabular}

In view of this one to one correspondence between these points and the real numbers, it is customary to say that the real numbers have only one dimension. As the establishment of a one to one correspondence between points and numbers has been so extremely fruitful in the development of mathematics, it was natural that common concepts relating to these fundamental notions should receive common names.

The points in the plane are commonly placed in a one to one correspondence with all possible pairs of real numbers by means of an arrangement in which the numbers represent the distances of the points from two fixed lines cutting each other at right 
angles. Such an arrangement is suggested by the following diagram:

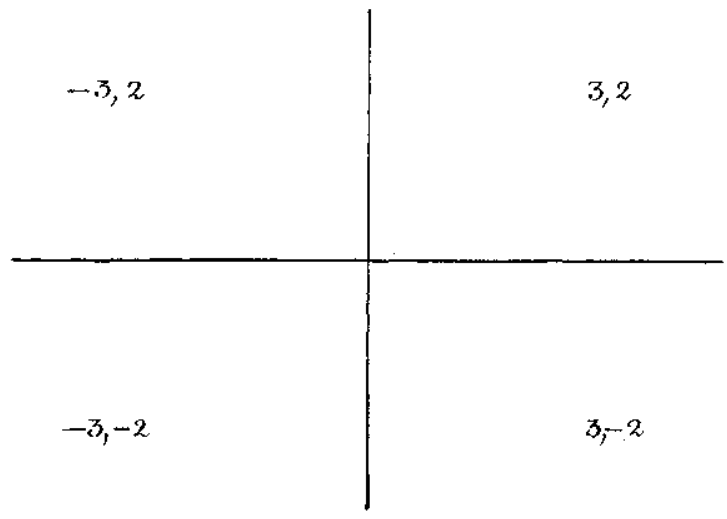

Fig. 2

Hence we say the totality of the pairs of real numbers has two dimensions. Every possible pair of real numbers may be uniquely represented by a line segment on a straight line if the first number of the pair represent the initial point of the segment and the second number represent the length of the segment, the sign of this number indicating the direction. For instance, the pairs $(-3,2),(3,-2)$ may be representer as follows:

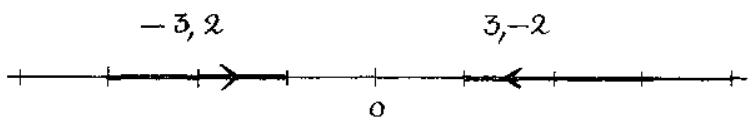

Fig. 3

Since the possible pairs of real numbers can be placed in a one to one correspondence both with the points in the plane and also with the line segments on a given line, it is natural to say that the number of line segments on a straight line is equivalent to the number of points in the plane. Since the plane is said to have two dimensions when the point is taken as the element, the line should be said to have two dimensions if the line segments are regarded as the elements. The fact which is to be emphasized in this connection is that the dimension of a geometric figure is not an intrinsic property of the figure but it depends upon what is regarded as the element.

By extending the notions mentioned above we may associate with each triplet of real numbers a point in space, and with each 
point in space a triplet of real numbers in such a way that there is a one to one correspondence between the triplets and the points of space. Hence the possible triplets are regarded as having three dimensions. There is a one to one correspendence between the possible circles in a plane and the triplets of real numbers if the first two numbers of the triplets indicate the centers of the circles while the last represents the length of the circumference, the sign indicating the direction in which the circumference is described. In view of this one to one correspondence between the points of space, the triplets of real numbers, and the circles in a plane, it is natural to regard the plane as having three dimensions if the elements are circles.

Just as the totality of sets of three real numbers is said to have three dimensions so the totality of sets of four real numbers is said to have four dimensions. Since a set of two real numbers represents a point in a plane, or a point in a two dimensional space, and a set of three real numbers represents a point in a three dimensional space, a set of four real numbers may be said to represent a point in a four dimensional space. It is easy to establish a one to one correspondence between the sets of four real numbers and some geometrical figures. When this is done the smallest space in which these figures lie is said to be four dimensional when the figures are regarded as the elements.

For instance, all the possible circles which are parallel to a given plane constitute a four dimensional manifoldness, since the centers of these circles may be uniquely represented by the first three, in the possible sets of four real numbers, while the last numbers in the sets may represent the length of the circumferences, the sign indicating the direction in which the circumferences are described. We thus obtain a one to one correspondence between the possible sets of four real numbers and the totality of parallel circles. We shall show that such a correspondence may also be established between these sets and the totality of the squares in a plane. Hence we shall say that the plane has four dimensions if all the possible squares lying in it are considered as the elements, while it has two or three dimensions, respectively, as the points or the circles are taken as the elements.

To exhibit clearly that there is a one to one correspondence between the totality of sets of four real numbers and the totality of squares lying in a plane, it seems desirable to emphasize the fact that by definition an infinite multitude is one in which a part 
is equivalent to the whole. That is, we can establish a one to one correspondence between the units of an infinite multitude and the units of a part of this multitude. Although this statement may appear strange at first it is nothing more than saying that the equation

$$
y+1=\frac{1}{x}
$$

can be solved uniquely for every positive value of $y$ and that two different values of $y$ always give rise to two different values of $x$. In other words, this equation establishes a one to one correspondence between the totality of positive numbers and the numbers representing the corresponding values of $x$. It is evident that the limits of $x$, when $y$ represents successively all the positive numbers, are $O$ and I. Hence we have to say that there is a one to one correspondence between all the positive numbers (including those from $O$ to $I$ ) and the numbers extending from o to ' 1 . By means of the equation.

$$
y+1=\frac{a}{x}
$$

we can similarly establish a one to one correspondence between the numbers from o to $a$ and all the positive numbers, if $a$ is any positive number whatever.

With this example before us it is easy to see that there is a one to one correspondence between the possible squares in the plane and the totality of sets of four real numbers. The first two numbers of such a set may represent the center of the square and the third may represent its perimeter, the sign indicating the direction in which the perimeter is described. The fourth numbers in all the possible sets may represent uniquely some angle from o to 90 made by a side of the square with a fixed line. To do this it is only necessary to establish a one to one correspondence between all the possible real numbers and the numbers from o to 90 . This may be done in many ways; for instance, we may establish a one to one correspondence between all the positive numbers $(y)$ and the numbers $(x)$ from o to 45 by means of the equation

$$
x=\frac{45}{y+1}
$$

The negative numbers may then be placed in a one to one correspondence with all the numbers from 45 to 90 by the rule that the number corresponding to a negative number is 45 plus the one corresponding to a positive number $(y)$ having the same absolute value. That is, we determine $x$ by means of the first 
or second of the following equations as $y$ is positive or negative:

$$
x=\frac{45}{y+1} \quad x=\frac{45}{1-y}+45
$$

The method of argument followed to exhibit that the plane has four dimensions if the squares are regarded as its elements may also be employed to show that space has four dimensions if the spheres are regarded as its elements. In fact, the first three numbers of every set of four real numbers may be regarded as the centers of spheres and the fourth numbers may be placed in a one to one correspondence with the positive numbers, and then these positive numbers may be used to represent the length of the radii of the possible spheres.

From the fact that there is a one to one correspondence between the points of a line and those of any finite line segment, it results that any line segment, besides the zero segment, is one dimensional if its points are regarded as its element and that it is two dimensional if its line segments are regarded as the elements. Similarly any arbitrary portion of the plane is four dimensional if the squares in it are regarded as its elements and an arbitrary portion of space is four dimensional if the totality of the spheres lying in it is regarded as constituting the elements. The fundamental idea of $n$ dimensions is a one to one correspondence between its elements and the totality of sets of $n$ real numbers. From this standpoint the fourth dimension has very much in common with the smaller number of dimensions and these common properties seem to offer the most fertile field of study. This conclusion is in accord with Poincaré's observations that "the great advances of the past were made by the union of two sciences, by observing similarity of form regardless of dissimilarity of matter, by modeling the one after the other."*

- In the process of utilizing the advances of one science in other related sciences it frequently has been helpful to assign to a term broader meaning. In fact, in the article to which reference has been made, Poincaré said that in a certain sense mathematics is the art of giving the same name to different things. The use of the term dimension for sets of numbers is in harmony with this fertile tendency which helps to clarify and systematize our mathematical knowledge. The term fourth dimension is used because it emphasizes the relation between the more complex and the simpler concepts. By observing these relations progress becomes easier and extensive developments present fewer difficulties. 